\title{
Compliance with NICE guidance on the use of anti-TNF $\alpha$ agents in ankylosing spondylitis: an East and West Midlands regional audit
}

\author{
Frances Rees, Gillian Peffers, Carolyn Bell, Karen Obrenovic, Ravinder Sandhu, Jonathan Packham \\ and Nicola Erb on behalf of West Midlands Regional STC and East Midlands Regional Audit Group
}

\begin{abstract}
Here we report on an audit performed to examine compliance with National Institute for Health and Clinical Excellence (NICE) guidelines for the use of anti-tumour necrosis factor alpha (anti-TNF $\alpha$ ) in treating patients with ankylosing spondylitis (AS). Data from 17 rheumatology centres across the Midlands were collected prospectively from patients with AS attending outpatient clinics and retrospectively in patients receiving anti-TNF $\alpha$ but not attending outpatient clinics during the audit. In total, $80 \%$ of the 416 patients for whom data were collected were male. Of the 238 patients recruited prospectively, $41 \%$ were receiving anti-TNF $\alpha$. Reviewing all patients on anti-TNF $\alpha(\mathrm{N}=275)$, pre-treatment assessments 12 weeks apart were documented in $55 \%$ of patients. After anti-TNF $\alpha$ treatment had started, regular 12-weekly assessments occurred in $46 \%$ of patients. Therefore, compliance with NICE guidance was found to vary among centres. Based on our audit, clinical capacity, and clinical or patient choice might be influencing the suboptimal adherence seen in assessment timing suggested by NICE guidelines relating to the use of anti-TNF $\alpha$ in treating patients with AS.
\end{abstract}

KEY WORDS: ankylosing spondylitis, anti-TNF $\alpha$, audit, NICE

\section{Introduction}

Ankylosing spondylitis (AS) is an inflammatory seronegative spondyloarthropathy of unknown aetiology. The prevalence of AS has been estimated to range from $0.05 \%$ to $0.23 \%$, with an annual incidence of 6.9 per $100,000 .{ }^{1}$ Historically, treatments available for this condition were physical therapies, nonsteroidal anti-inflammatory drugs (NSAIDs) and diseasemodifying anti-rheumatic agents that, although effective on peripheral joint involvement, have little effect on spinal

Frances Rees, ${ }^{1}$ rheumatology specialty registrar; Gillian Peffers, ${ }^{2}$ rheumatology specialty registrar; Carolyn Bell, ${ }^{3}$ rheumatology specialty registrar; Karen Obrenovic, ${ }^{4}$ clinical audit manager; Ravinder Sandhu, ${ }^{4}$ consultant rheumatologist; Jonathan Packham, ${ }^{5}$ consultant rheumatologist; Nicola Erb, ${ }^{4}$ consultant rheumatologist

${ }^{1}$ Nottingham University Hospitals NHS Trust, Nottingham; ${ }^{2}$ Heart of England NHS Trust, Birmingham; ${ }^{3}$ Worcestershire Acute NHS Trust, Worcester; ${ }^{4}$ The Dudley Group NHS Foundation Trust, Dudley; ${ }^{5}$ University Hospital of North Staffordshire, Stoke-on-Trent disease. ${ }^{2}$ These treatments provide symptomatic relief, but few have any influence on disease progression. In 2008, the National Institute for Health and Clinical Excellence (NICE) published guidance on the use of anti-tumour necrosis factor alpha (anti$\mathrm{TNF} \alpha$ ) agents in severe active AS. ${ }^{1}$ These guidelines followed evidence from randomised controlled trials (RCTs) that had demonstrated a statistically significant improvement in disease activity with etanercept, ${ }^{3-6}$ adalimumab $^{7}$ and infliximab ${ }^{8}$ compared with placebo. At the time that the audit was performed, only etanercept and adalimumab were approved by NICE. Golimumab has been approved subsequently. ${ }^{9}$ NICE recommended that these drugs were only used in patients who fulfilled the Modified New York criteria for AS (Box 1) ${ }^{10}$ and had active disease defined by a Bath Ankylosing Spondylitis Disease Activity Index (BASDAI) ${ }^{11}$ Score $\geqslant 4$ units and a spinal pain visual analogue score (VAS) $\geqslant 4$ units (on a scale of $0-10$ ) on two occasions at least 12 weeks apart. To qualify, patients should not have responded to two different NSAIDs taken at the maximum tolerated dose for at least four weeks. Once a patient has started treatment, continuing efficacy must be evaluated every 12 weeks. Treatment should only be continued in the presence of an adequate response as defined in the NICE guidance ${ }^{1}$ as a reduction of the BASDAI score by $50 \%$ or $\geqslant 2$ units and a reduction in spinal pain VAS of $\geqslant 2$ units.

The aim of this regional audit was to evaluate the prescribing practice of anti-TNF $\alpha$ agents in patients with AS in secondary care and compare this to NICE guidance.

\section{Methods}

\section{Study design}

Seventeen secondary-care rheumatology units across the East and West Midlands were recruited (Box 2). Units were invited to collect data in two arms: prospective and retrospective. Prospective data were collected during a four-week period in July 2010 from all patients with a diagnosis of AS attending general or specialised rheumatology outpatient clinics. Retrospective data were collected from case-note review of randomly chosen patients with AS already taking an anti-TNF $\alpha$ agent but not attending for follow-up during the observation period. The retrospective arm was included to obtain an adequate number of patients to assess selection suitability and monitoring of anti-TNF $\alpha$ treatment once initiated. 


\section{Proforma design}

A proforma was developed to collect data on patient demographics, pattern of disease (axial (spine +/- hip), mainly peripheral, or both axial and peripheral), presence of associated human leucocyte antigen (HLA)-B27 conditions and past or present anti-TNF $\alpha$ use, BASDAI and spinal pain VAS score. The proforma incorporated the audit criteria outlined in the NICE Technology Appraisal $143^{12}$ for patients currently or previously taking anti-TNF $\alpha$, including whether they met the modified New York criteria. The form was piloted in two participating units and, following minor modifications, was distributed to all units. Written instructions on data collection were sent out in advance of the proformas.

\section{Data analysis}

Proformas were returned to a central unit and scanned. Frequencies and percentages were calculated using SPSS version 14 .

\section{Results}

In total, 416 proformas were completed: 238 prospectively and 178 retrospectively. Of these patients, $93.2 \%$ were aged between 25 and 75 years, with a gender ratio of 4 males to 1 female. All patients met the modified New York criteria for AS.

\section{Pattern of spondylitis and associated diseases}

In total, 27\% patients (113/416) had mixed axial and peripheral joint involvement, 1\% (5/416) exhibited mainly peripheral joint disease and 69\% (287/416) had only axial disease. In 3\% (11/416) no pattern was assigned.

Extra-articular manifestations were present in $38 \%$ of patients. In total, $25 \%$ had uveitis, (105/416), $8 \%$ had psoriasis (32/416) and $9 \%(38 / 416)$ had inflammatory bowel disease

\section{Suitability for, and distribution of, anti-TNF $\alpha$ treatment according to NICE}

In total, $41 \%$ (97/238) of patients assessed prospectively were currently receiving anti-TNF $\alpha$ treatment: 51\% (49/97) were receiving etanercept, 45\% (44/97) adalimumab and 4\% (4/97) infliximab. Of the patients seen prospectively, $5 \%(11 / 238)$ had previously been taking anti-TNF $\alpha$. Of those patients who had never received anti-TNF $\alpha$ treatment, 45\% (58/130) currently fulfilled NICE eligibility criteria. Of these, $38 \%(22 / 58)$ were currently being assessed for starting treatment, $24 \%(14 / 58)$ had already declined treatment, $19 \%(11 / 58)$ had recognised contraindications to treatment, $1.7 \%$ (1/58) were having funding problems and $17 \%$ $(10 / 58)$ were not treated on clinician's judgement.

Evaluating all patients currently on anti-TNF $\alpha$ agents (178 retrospective and 97 prospective), 56\% (154/275) had pretreatment assessments documented in the notes at least 12 weeks apart (consistent with NICE guidance). A further 26\% (73/275)
Box 1. Modified New York criteria for diagnosis of ankylosing spondylitis.

A definite diagnosis of ankylosing spondylitis (AS) requires the radiological criterion and at least one clinical criterion to be satisfied, as defined below.

Radiological criterion

Sacroiliitis at least grade 2 bilaterally or grade 3 or 4 unilaterally. Clinical criteria

- Low back pain and stiffness for more than three months that improves with exercise but is not relieved by rest.

- Limitation of motion of the lumbar spine in both the sagittal and frontal planes.

- Limitation of chest expansion relative to normal values correlated for age and sex.

All reasonable measures should be taken to ensure that symptoms are the result predominantly of AS and that alternative causes, including spinal fracture, disc disease and fibromyalgia, are excluded.

\section{Box 2. Participating rheumatology units.}

Birmingham City Hospital/Sandwell

Cannock Chase Hospital

Haywood Hospital, Stoke-on-Trent

The Dudley Group

Hereford County Hospital

Kings Mill Hospital, Mansfield

Leicester Royal Infirmary

Lincoln County Hospital

New Cross Hospital, Wolverhampton

Queen's Hospital, Burton

Queen's Medical Centre, Nottingham

Royal Derby Hospital

Solihull Hospital

University Hospital Birmingham

University Hospital Coventry

Warwick Hospital

Worcestershire Royal Hospital

had these documented at a four-week interval, consistent with previous British Society of Rheumatology (BSR) guidance. ${ }^{13}$ Previous treatment with two or more NSAIDs was documented in $90 \%(249 / 275)$ of patients.

\section{Monitoring and withdrawal of treatment}

NICE guidelines stipulate that, 12 weeks after starting antiTNF $\alpha$ treatment, a further BASDAI and spinal pain VAS should be carried out in all cases to assess primary response. If this response is deemed inadequate, then a further assessment after a six-week interval should be performed. If patients do not show treatment response at this point, then the anti-TNF $\alpha$ treatment should be withdrawn. In this audit, the first assessment was recorded at 12 weeks in 59\% (162/275) of cases. This varied 
between units from $9 \%$ to $100 \%$ of their cases, with a median of $57 \%$ (interquartile range $39-85 \%$ ). An additional $22 \%$ of the total cases were assessed between four and six months after starting anti-TNF $\alpha$ treatment. However, $11 \%$ had their first assessment more than six months after commencement. In $8 \%$, no time interval was recorded.

Of the first assessments, 17\% (48/275) showed an inadequate treatment response (ie not demonstrating the required reduction in BASDAI and spinal pain VAS). Of these, only 19\% (9/48) had treatment discontinued. Data collected were not sufficiently detailed to ascertain the proportion of patients showing an adequate response at a further six-week assessment.

Of all patients currently taking anti-TNF $\alpha$ agents, $46 \%$ $(128 / 275)$ had regular 12 -weekly assessments documented in their notes. This ranged from $8 \%$ to $100 \%$ between units, with a median of $44 \%$ (interquartile range $21.5-78.0 \%$ ).

\section{Reasons for switching or discontinuation of agents}

Of the 275 patients currently taking anti-TNF $\alpha$ agents, $13 \%$ $(35 / 275)$ were on a second or subsequent agent. In $43 \%$ of these cases $(15 / 35)$ this was because of an adverse event with a previous agent. In $26 \%(9 / 35)$, it was because of a secondary loss of effect and, in $17 \%(6 / 35)$, it was because of an initial inadequate response. In $11 \%(4 / 35)$ of cases it was because of a switch from infliximab to an alternative agent following the publication of NICE TA 143 in May 2008. In one case no reason was documented.

In total, 19 patients who had previously been treated with anti-TNF $\alpha$ had stopped biological therapy completely. This was because of: an adverse event in 42\% (8/19); an initial inadequate response not followed by a switch to an alternative agent in $32 \%$ $(6 / 19)$; a secondary loss of effect in $10.5 \%(2 / 19)$; and for other reasons in $16 \%(3 / 19$; joint replacement surgery (1), patient wishing to stop because of personal funding difficulties (1); and lack of active inflammation on MRI off treatment (1)).

\section{Adverse events}

In total, 31 patients (11\%) had an adverse event while taking anti-TNF $\alpha$. In 12 patients (39\%) this occurred at less than three months after starting treatment and, in 19 patients $(61 \%)$, it occurred after three months. This resulted in treatment cessation in nine patients, nine patients continued treatment, 12 patients switched anti-TNF $\alpha$ and, in one patient, the outcome was unknown.

\section{Discussion}

Good adherence to NICE guidance was observed in patient selection and choice of biological agent. However, there were several areas of suboptimal adherence particularly related to the timing of assessments and monitoring of therapy. Only 56\% of initial pretreatment assessments met the current NICE recommended interval of at least 12 weeks, with a further $26 \%$ being assessed according to previous guidelines set out by the BSR. ${ }^{13}$ This sug- gests that a difference between guidelines has led to some confusion in clinical practice. Less than half of patients received the recommended 12-weekly follow up assessments post anti-TNF $\alpha$ treatment initiation. This significant shortfall might be because of: local financial, staffing or clinic capacity issues, thus highlighting the need for adequate service provision in secondary care rheumatology departments prescribing anti-TNF $\alpha$ for AS; clinicians might feel that 12-weekly assessments are too frequent, given that assessments for rheumatoid arthritis (RA) are performed every six months; ${ }^{14}$ or patients responding well to antiTNF $\alpha$ might not wish to attend clinic appointments every 12 weeks. The wide variation in adherence to the guidelines between the 17 different units suggests that some clinicians view the technology appraisals as guidelines rather than strict criteria.

There are no previous publications reviewing adherence to the NICE criteria for the use of anti-TNF $\alpha$ in AS.

Limitations to the study included prospective collection of data from consecutive patients attending secondary-care rheumatology units, introducing selection bias, particularly regarding disease severity. Patients would be more likely to attend the outpatient clinic if they were having a disease flare or if they were having an assessment for anti-TNF $\alpha$. Therefore, it is likely that the relatively high percentage of prospective patients currently taking anti-TNF $\alpha(41 \%)$ is an overestimate. While clinicians were completing the proformas, they might also have noticed that the patient qualified for treatment, increasing the proportion of patients 'currently being assessed for starting treatment'. Physicians indicated that all patients taking antiTNF $\alpha$ met the modified New York criteria; however, no supporting evidence was collected as part of the proforma. Data were collected on patients' most recent BASDAI and/or pain VAS score, and compliance with BASDAI and/or pain VAS NICE criteria in patients at anti-TNF $\alpha$ initiation, rather than collecting all BASDAI and/or pain VAS scores over time. These data would have enhanced the audit evaluation, but it was felt to be too onerous for clinicians to complete such a collection in a clinical setting. The proforma design did not enable assessment of the six-week review after an inadequate response to anti-TNF $\alpha$ after 12 weeks. Consequently, it was not possible to draw accurate conclusions regarding the high number of patients continuing on treatment without an adequate response. However, one reason might be that the patients had experienced some symptomatic improvement despite not complying with the NICE criteria for treatment response.

Further work should look at the number of patients with supporting evidence for meeting the modified New York criteria. The number of non-responders having a six-week follow-up could be explored and, if treatment continues, to examine the reasons for this. Evaluation of the reasons why 12-weekly assessments were not occurring would be of value, as would evaluating the clinical and financial impact of performing six-monthly response assessments instead of the current 12-weekly assessments in anti-TNF $\alpha$ responders, given that there is evidence to suggest that remission in responders is maintained for up to at least two years. ${ }^{15}$ 
In conclusion, our audit highlights some areas of good adherence to the NICE guidelines and some areas for improvement. Variation between national NICE and/or BSR, and AS and/or RA guidelines might have caused some confusion among clinicians over when to assess for response. An evaluation of the impact of the current common practice of performing six-monthly response assessments, compared with the current NICE guideline advice of 12-weekly assessments in anti-TNF $\alpha$ responders should be considered.

\section{Acknowledgements}

We are grateful to all individuals at participating units who collected data within the West Midlands regional STC and East Midlands regional audit group, and to the Healthcare Quality Improvement Partnership (HQIP), who provided financial support administrative costs. There are no conflicts of interest.

\section{References}

1 National Institute for Health and Clinical Excellence. Adalimumab, etanercept and infliximab for ankylosing spondylitis. NICE technology appraisal guidance 143. London: NICE, 2008.

2 Clegg DO, Reda DJ, Abdellatif M. Comparison of sulfasalazine and placebo for the treatment of axial and peripheral articular manifestations of the seronegative spondylarthropathies: a Department of Veterans Affairs cooperative study. Arthritis Rheum 1999;42:2325-9.

3 Gorman JD, Sack KE, Davis JC Jr. Treatment of ankylosing spondylitis by inhibition of tumor necrosis factor alpha. $N$ Engl J Med 2002;346:1349-56.

4 Davis JC Jr, van der Heijde D, Braun J et al. Recombinant human tumor necrosis factor receptor (etanercept) for treating ankylosing spondylitis: a randomized, controlled trial. Arthritis Rheum 2003;48:3230-6.

5 Brandt J, Khariouzov A, Listing J et al. Six-month results of a doubleblind, placebo-controlled trial of etanercept treatment in patients with active ankylosing spondylitis. Arthritis Rheum 2003;48:1667-75.
6 Calin A, Dijkmans BA, Emery P et al. Outcomes of a multicentre randomised clinical trial of etanercept to treat ankylosing spondylitis. Ann Rheum Dis 2004;63:1594-600.

7 van der Heijde D, Kivitz A, Schiff $\mathrm{MH}$ et al. Efficacy and safety of adalimumab in patients with ankylosing spondylitis: results of a multicenter, randomized, double-blind, placebo-controlled trial (ATLAS). Arthritis Rheum 2006; 54:2136-46.

8 van der Heijde D, Dijkmans B, Geusens P et al. Efficacy and safety of infliximab in patients with ankylosing spondylitis: results of a randomized, placebo-controlled trial (ASSERT). Arthritis Rheum 2005;52:582-91.

9 National Institute for Health and Clinical Excellence. Golimumab for the treatment of ankylosing spondylitis. NICE technology appraisal guidance 233. London: NICE, 2001.

10 Van der Linden S, Valkenburg HA, Cats A. Evaluation of diagnostic criteria for ankylosing spondylitis: a proposal for modification of the New York criteria. Arthritis Rheum 1984;27:361-368.

11 Garrett S, Jenkinson T, Kennedy LG et al. A new approach to defining disease status in ankylosing spondylitis: the Bath Ankylosing Spondylitis Disease Activity Index. Rheumatology 1994;21:2286-91.

12 National Institute for Health and Clinical Excellence. Ankylosing spondylitis - adalimumab, etanercept and infliximab: audit support. London: NICE, 2008.

13 Keat A, Barkham N, Bhalla A et al. BSR guidelines for prescribing TNF- $\alpha$ blockers in adults with ankylosing spondylitis. Report of a working party of the British Society for Rheumatology. Rheumatology 2005;44:939-47.

14 National Institute for Health and Clinical Excellence. Adalimumab, etanercept and infliximab for the treatment of rheumatoid arthritis. NICE technology appraisal guidance 130. London: NICE, 2007.

15 van der Heijde D, Schiff MH, Sieper J et al. Adalimumab effectiveness for the treatment of ankylosing spondylitis is maintained for up to 2 years: long-term results from the ATLAS trial. Ann Rheum Dis. 2009;68:922-9.

Address for correspondence: Dr F. Rees, Nottingham University Hospitals NHS Trust, Derby Road, Nottingham, NG7 2UH.

Email: frees@doctors.org.uk 